\title{
Synthesis of N-methylthieno [2, 3-d] Pyrimidin-4-amine Thienopyrimidine
}

\author{
Huajun Lei ${ }^{1}$, Min Wang ${ }^{1}$, Jiaqian Han ${ }^{1}$, and Zhou Lan ${ }^{1, a}$ \\ ${ }^{1}$ School of Pharmacy, Jiangxi Science \& Technology Normal University, Nanchang 330013, China \\ "alan8709@yeah.net
}

Keywords: Thieno [2,3-d] pyrimidine; Phosphoinositide 3-kinase; Synthesis

\begin{abstract}
Thieno[2,3- $d$ ]pyrimidine is a key intermediate in many biologically active compounds such as GDC-0941 and BKM 120. In this work, a rapid synthetic method for compound (4) was established. The compound (4) was synthesized from the commercially available methyl 2aminothiophene-3-carboxylate and formimidamide through three steps including condensation reaction, chlorination, and nucleophilic substitution. The structure was confirmed by MS and ${ }^{1} \mathrm{H}$ NMR. Furthermore, the total yield of the three steps was $54 \%$.
\end{abstract}

\section{Introduction}

Cancer is a complex, widespread, and awful disease. That is a group of different diseases character-

Idea by un-controlled cellular growth, tissue damage, and invasion [1]. In 2008, there are 7.6 million people having died of cancer. Nowadays there are many ways deal with cancer; one of the major treatment of cancer is chemotherapy. The anti-tumor drugs not only kill the cancer cells but will also damage the normal cells. Therefore development of a selective inhibition of cancer cell growth of the drug becomes necessary. Many articles reported that cancer is related to the phosphoinositide 3-kinase (PI3K)/AKT/mammalian target of rapamycin (mTOR) signaling pathway and normal cells changing. The PI3K pathway is also a common pathway that is activated at abnormally high levels in various human cancers. In recent years, there are many literature had been reported that thienopyrimidine derivatives show a wide variety of biological activities, for example, as an antibacterial and antihypertensive agents, as pesticides, and also as herbicides, even as plant growth regulators[2]. The phosphoinositide 3-kinase (PI3K)/AKT/the mammalian target of rapamycin (mTOR) signal transduction pathway is involved in many essential cellular functions including cell growth, differentiation, proliferation, survival, motility, and intracellular trafficking[3-4]. Thienopyrimidine derivatives were demonstrated to bear nanomolar PI3K $\alpha$ inhibitory potency with over 100-fold selectivity against mTOR kinase[5]. Up to now, there is few literature report that how to synthesis thieno[2,3-d]pyrimidine. In order to obtain the target compound, it was synthesized throught three steps, using methyl 2-aminothiophene-3-carboxylate and formamidine acetate as a starting material, including condensation reaction, chlorination and nucleophilic substitution. Most of the synthetic methods of thieno[2,3-d]pyrimidine which reported in the literature have the drawbacks such as long synthetic route, harmful to environment and low yield. Therefore, the optimization of the synthetic route and methods of thieno[2,3-d]pyrimidine is necessary. At the same times, some literature have reported that the thienopyrimidine core can be used in preparing potent P2Y12 inhibitors[6]. For example, GDC-0941 and BKM 120 are a selective PI3K inhibitors which have been advanced into clinical evaluation[7-10]. At the same times, some literature point out 2-substituted thienopirimidin-4-one derivatives may serve as novel spasmolytic agents to treat disorders of smooth muscle function, and those compounds have a common features that are thieno [2,3-d] pyrimidin[11], the structure of thieno[2,3-d]pyrimidin and target compound was shown in Fig. 1.

In this study, we designed and optimized the synthetic methods for $N$-methylthieno[2,3$d$ ]pyrimidin-4-amine (4) and made it more suitable for industrial production. 
<smiles></smiles>

Thieno[2,3- $d]$ pyrimidin-4-one

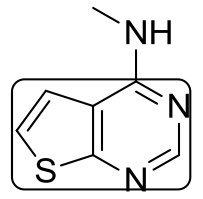

Target compound

Figure 1. Structure of thieno[2,3- $d]$ pyrimidin-4-one and target compound

\section{Materials and Methods}

All the chemicals and reagents used in current study were of analytical grade. NMR spectra were performed using Bruker $300 \mathrm{MHz}$ spectrometers (Bruker Bioscience, Billerica, MA, USA) with TMS as an internal standard. Mass spectra (MS) were taken in ESI mode on Agilent 1100 LC-MS (Agilent, Palo Alto, CA, USA). Elemental analysis was determined on a Carlo-Erba 1106 Elemental analysis instrument (Carlo Erba, Milan, Italy). All the materials obtained from commercial suppliers and used without purification, unless otherwise specified. Yields were not optimized. TLC analysis was carried out on silica gel plates GF254 (Qindao Haiyang Chemical, China).

\section{Synthesis of Compounds}

The structures and the synthetic route were shown in Scheme 1.<smiles>CNc1ncnc2sc(C(C)C)cc12</smiles>

Scheme 1. The synthetic route of compound 4

Reagents and Conditions: (a) formamidine acetate, ethanol, $78{ }^{\circ} \mathrm{C}, 15 \mathrm{~h}$. (b) phosphoryl trichloride, toluene, $110^{\circ} \mathrm{C}, 1 \sim 2 \mathrm{~h}$. (c) $30 \%$ methanamine, DMF, $155^{\circ} \mathrm{C}, 8 \mathrm{~h}$.

Thieno [2,3-d] Pyrimidin-4-ol (2). Methyl 2-aminothiophene-3-carboxylate (1) (30 g, 190 $\mathrm{mmol})$ and formamidine acetate $(25.8 \mathrm{~g}, 280 \mathrm{mmol})$ were added in three-necked flask which contain ethanol $(50 \mathrm{~mL})$, then stirred and refluxed at $78{ }^{\circ} \mathrm{C}$ for $3 \mathrm{~h}$. After the reaction completed, the mixture was poured into ice-cold water. The solid product formed was filtered and crystallized from ethanol. Yield 60\%. ${ }^{1} \mathrm{H}$ NMR (400 MHz, DMSO) $\delta 12.51(\mathrm{~s}, 1 \mathrm{H}), 8.15(\mathrm{~s}, 1 \mathrm{H}), 7.60(\mathrm{~d}, J=$ $0.9 \mathrm{~Hz}, 1 \mathrm{H}), 7.42$ (dd, $J=5.8,1.4 \mathrm{~Hz}, 1 \mathrm{H}) . \mathrm{MS}(\mathrm{ESI}): \mathrm{m} / \mathrm{z} 153.0[\mathrm{M}+\mathrm{H}]^{+}$.

4-Chlorothieno [2,3- $d$ ] Pyrimidine (3). Thieno[2,3-d]pyrimidin-4-ol (2) (1 g, $6.6 \mathrm{mmol})$ was suspended in toluene $(5 \mathrm{~mL})$, then added the phosphoryl trichloride $(10 \mathrm{~mL})$ in the mixture, then at the $110{ }^{\circ} \mathrm{C}$ refluxed for $1 \sim 2 \mathrm{~h}$. Using the TLC monitoring, after the reaction completed, the mixture was slowly poured into ice-cold water and was stirred at room temperature. The solid product formed was filtered, washed with ice-cold water and dried and crystallized from ethanol. Yield 90\%, mp: 97-98 ${ }^{\circ} \mathrm{C} .{ }^{1} \mathrm{H}$ NMR (400 MHz, DMSO) $\delta 8.95(\mathrm{~s}, 1 \mathrm{H}), 8.14(\mathrm{~d}, J=6.0 \mathrm{~Hz}, 1 \mathrm{H})$, $7.58(\mathrm{~d}, J=6.0 \mathrm{~Hz}, 1 \mathrm{H})$. MS (ESI): $\mathrm{m} / \mathrm{z} 171.9[\mathrm{M}+\mathrm{H}]^{+}$.

$N$-methylthieno [2,3-d] Pyrimidin-4-Amine. 4-Chlorothieno [2,3- $d$ ]pyrimidine(0.46 g, 3 mmol) (3) and $30 \%$ methanamine $(0.2 \mathrm{~mL}, 30 \mathrm{mmol})$ were dissolved in $N, N$-dimethylformamide (DMF) at 
$155{ }^{\circ} \mathrm{C}$ for $3 \mathrm{~h}$. After the reaction completed, cooling to room temperature, the target compound was extracted with dichloromethane(DCM) and water from the mixture, dried over anhydrous $\mathrm{Na}_{2} \mathrm{SO}_{4}$, filtered and evaporated, then to give $N$-methylthieno[2,3- $d$ ]pyrimidin-4-amine (4) in 54\% yield, mp: $105-107{ }^{\circ} \mathrm{C} .{ }^{1} \mathrm{H}$ NMR (400 MHz, DMSO) $\delta 8.35$ (s, 1H), 7.99 (s, 1H), 7.55 (s, 1H), 2.97 (s, 3H). MS (ESI): m/z 166.0[M+H ${ }^{+}$.

\section{Conclusions}

In conclusion, N-methylthieno[2,3-d]pyrimidin-4-amine (4) was synthesized from the commercially available methyl 2-aminothiophene-3-carboxylate (1) and formamidine acetate through three steps including condensation reaction, chlorination, nucleophilic substitution. The synthetic method of compound (4) and the reactions conditions were optimized, the purity of the product is high. All the compounds structure was confirmed by MS and 1H NMR spectrum.

\section{Acknowledgments}

We gratefully acknowledge the generous support provided by Project supported by Doctoral Scientific Research Foundation of Jiangxi Science \& Technology Normal University and Program of Key Laboratory of Drug Design and Optimization, Jiangxi Science \& Technology Normal University (300098010306) and College Students' Science and Technology Innovation Project of Jiangxi Province.

\section{References}

[1] Wei L.; Malhotra S V. Synthesis and cytotoxicity evaluation of novel pyrido [3, 4- $d$ ] pyrimidine derivatives as potential anticancer agents[J]. Med. Chem. Comm., 2012, 3(10): 1250-1257.

[2] Birgit W.; Stefan F.; Norbert K. A practical synthesis of 5-functionalizedthieno[2,3d]pyrimidines[J]. Tetrahedron Lett., 2015, 05: 104.

[3] Engelman J. A.; Luo J.; Cantley L. C. The evolution of phosphatidylinositol 3-kinases as regulators of growth and metabolism[J]. Nat. Rev. Genet., 2006, 7: 606-619.

[4] Liu P. X.; Cheng H. L.; Roberts T. M.; et al. Targeting the phosphoinositide 3-kinase pathway in cancer[J].Nat. Rev. Drug Discovery, 2009, 8: 627-644.

[5] Fangbin H.; Songwen L.; Heng X. Discovery of a Novel Series of Thienopyrimidine as Highly Potent and Selective PI3K Inhibitors[J]. ACS Med. Chem. Lett., 2015, 6(4): 434-438.

[6] Steven W. K. ; Rhonda M. L.; Barbara A. S.; et al. Thienopyrimidine-based P2Y12 platelet aggregation inhibitors[J]. Bioorg. Med. Chem. Let., 2009, 19: 5919-5923.

[7] Knight S. D.; Adams N. D.; Burgess J. L.; et al. Discovery of GSK2126458, a highly potent inhibitor of PI3K and the mammalian target of rapamycin[J]. ACS Med. Chem. Lett., 2010, 1: 39-43.

[8] Venkatesan A. M.; Dehnhardt C. M.; Delos Santos E.; et al. Bis(morpholino-1,3,5-triazine) derivatives: potent adenosine 5'-triphosphate competitive phosphatidylinositol-3kinase/mammalian target of rapamycin inhibitors: discovery of compound 26 (PKI-587), a highly efficacious dual inhibitor[J]. Med. Chem., 2010, 53: 2636-2645.

[9] Folkes A. J.; Ahmadi K.; Alderton W. K.; et al. The identification of 2-(1H-indazol-4-yl)-6-(4methanesulfonyl-piperazin-1-ylmethyl)-4-morpholin-4-yl-thieno[3,2-d]pyrimidine (GDC-0941) as a potent, selective, orally bioavailable inhibitor of class I PI3 kinase for the treatment of cancer[J]. Med. Chem., 2008, 51: 5522-5532.

[10]Burger M. T.; Pecchi S.; Wagman A.; et al. Identification of NVP-BKM120 as a potent, 
selective, orally bioavailable Class I PI3 kinase inhibitor for treating cancer[J]. ACS Med. Chem. Lett., 2011, 2: 774-779.

[11]Natale A. S.; Orazio P.; Ennio B.; et al. Synthesis and spasmolytic action of 2-substituted thienopyrimidin-4-one derivatives[J]. J. Pharm. Pharmacol., 2002, 54: 717-728. 